\title{
Estructura Nasal en Pacientes con Deformidad Facial Clase III
}

\author{
Structure of the Nose in Patients with Class III Facial Deformities
}

Sergio Olate ${ }^{*, * *}$; Mario Cantín*; Bélgica Vásquez ${ }^{* * * *}$ \& Gabriela Olate**

OLATE, S.; CANTÍN, M.; VÁSQUEZ, B. \& OLATE, G. Estructura Nasal en Pacientes con Deformidad Facial Clase III. Int. J. Morphol., 32(1):334-338, 2014.

RESUMEN: El objetivo de esta investigación fue conocer aspectos morfológicos de la estructura nasal en sujetos con diagnóstico de deformidad facial clase III. Se realizó un estudio con tomografía computadorizada Cone Beam sobre 20 pacientes entre 18 y 35 años para identificar mediciones a nivel de la apertura piriforme, huesos propios de la nariz y ángulos de dorso nasal y labio superior. El estudio se realizó en el software del sistema realizando las mediciones de acuerdo a condicione estándar; el análisis de datos fue de forma descriptiva y mediante la prueba $t$ de student considerando el valor de $p<0,05$ para obtener significancia estadística. Los resultados demostraron importantes variaciones en la altura de los huesos nasales y la forma de la apertura piriforme; las mediciones mostraron algunas variaciones donde el ángulo nasolabial fue el que presentó valores bajo los $104^{\circ}$, considerados fuera de la normalidad; no se presentaron relaciones significativa en ninguna de las evaluaciones realizadas en esta muestra. Se puede concluir que existen importantes variaciones anatómicas y que nuevas mediciones deben ser evaluadas para utilizarlas en métodos de diagnóstico y tratamiento.

PALABRAS CLAVE: Deformidad facial; Nariz; Morfometría facial.

\section{INTRODUCCIÓN}

La nariz y el mentón son elementos anatómicos unitarios de alto valor estético en la arquitectura facial. Esto hace que la rinoplastia cosmética se encuentre dentro de las cirugías más comúnmente realizadas para mejorar las características faciales; así, el conocimiento de la morfología nasal y sus variaciones son fundamentales para establecer condiciones basales y tratamientos quirúrgicos (Çakir et al., 2013).

Tanto la nariz como el mentón se deben encontrar dentro de una línea mediana facial, lo que en normalidad permite realizar una separación simétrica (o con alteraciones clínicamente no significativas) de ambas mitades del rostro lo que conlleva a que desvíos o alteraciones de forma, sean muy relevantes en el análisis de la estética facial (Bertossi et al., 2013).

La anatomía normal de la nariz implica grandes variaciones tanto en tejidos óseos como cartilaginosos (Begg \& Harkness, 1995), lo que hace complejo su manejo. Deformidades faciales como el prognatismo o la retrognatia maxilar son altamente asociadas con alteraciones en la forma nasal (Saeh et al., 2012), de manera que establecer la condición anatómica de estos sujetos es fundamental en la planificación y predicción del tratamiento quirúrgico.

La deformidad facial clase III (DF-III) presenta una deficiencia en el desarrollo del maxilar, la apertura piriforme y sus estructuras asociadas (Saeh et al.). Por esto, es posible que la DF-III presente condiciones y características estructurales específicas a nivel de nariz. El objetivo de esta investigación fue conocer algunos aspectos de la morfología nasal en sujetos con DF-III y definir características aplicables a la planificación quirúrgica de estos casos.

\section{MATERIAL Y MÉTODO}

La muestra analizada consistió en 20 pacientes adultos de ambos sexos, con edades entre 18 y 35 años. Se incluyeron pacientes con diagnostico de DF-III y alteraciones dentarias que consultaron por tratamiento de cirugía ortognática o corrección facial en la División de Cirugía Oral y Maxilofacial de la Universidad de La Frontera, Temuco, Chile. Los pacientes que presentaran cirugías faciales pre-

* División de Cirugía Oral y Maxilofacial, CIMA, Depto. Odontología Integral Adultos, Facultad de Odontología, Universidad de La Frontera, Temuco, Chile.

*** Centro de Investigación en Ciencias Biomédicas, Universidad Autónoma de Chile, Temuco, Chile.

*** Universidad de Tarapacá, Arica, Chile. 
vias de tejidos duros o blandos (i.e. rinoplastía), trauma facial previo o alteraciones morfológicas atribuidas a malformaciones faciales o corporales por defectos congénitos o síndromes fueron excluidos. También se excluyeron los pacientes que presentaron asimetrías faciales con más de $4 \mathrm{~mm}$ de desvió a la línea mediana facial. Todos los sujetos accedieron voluntariamente al estudio, firmando un consentimiento informado para la investigación.

Luego de la consulta inicial, se realizó el análisis clínico facial a todos los sujetos en condiciones secuenciales según el protocolo vigente en la División de Cirugía Oral y Maxilofacial. Fueron sometidos al estudio imagenológico, el cual incluyó la tomografía computadorizada Cone Beam (Pax Zenith, Vatech, Korea, 2011), utilizando 90 kV y 120 mA; la posición del sujeto y la captura de la imagen fue realizada por un técnico con experiencia previamente calibrado en la toma de imágenes de sujetos con deformidades faciales.

Las imágenes digitales fueron analizadas mediante el software Ez 3D 2009, donde se trazaron las medidas que se observan en las Figuras 1, 2 y 3, evidenciándose el ancho máximo superior e inferior de la apertura piriforme, la dimensión vertical de la apertura piriforme en el punto mediano entre los huesos nasales (HN) y espina nasal anterior (ENA), dimensión vertical de los HN, ancho del maxilar entre los procesos cigomáticos, distancia antero-posterior del maxilar en el plano mediano sagital, además de los ángulos que conforman la estructura nasal en los $\mathrm{HN}$, los tejidos blandos y a nivel nasolabial. Todas las medidas fueron realizadas y analizadas sobre la reconstrucción volumétrica y lateral bidimensional. Se consideró la imagen con mayor representatividad para la medición necesaria.

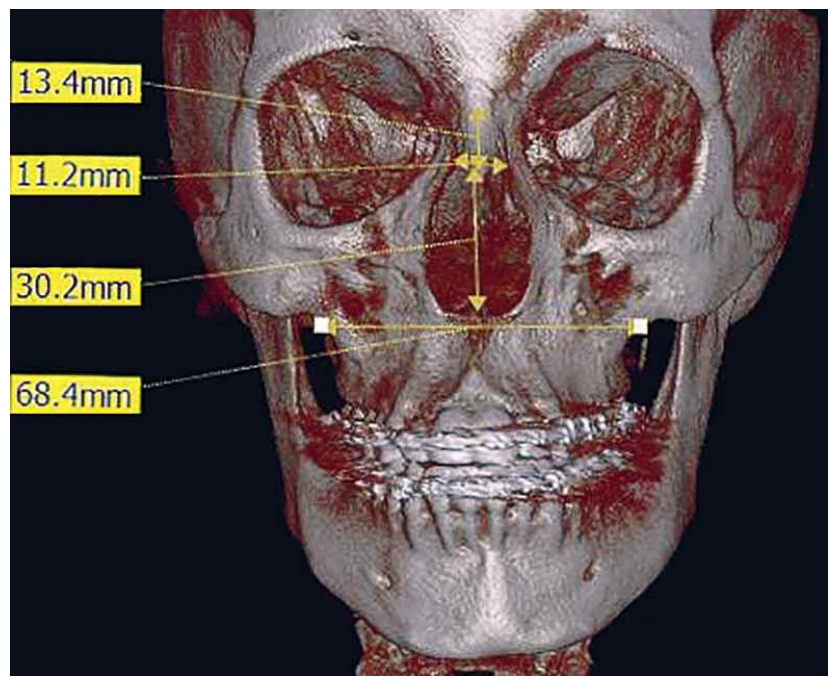

Fig. 1. Relaciones morfométricas obtenidas a partir de la reconstrucción esqueletal mediante TC cone beam de individuos con deformidad facial clase III.
Las mediciones fueron expresadas en milímetros o grados, según corresponda, y se realizaron en 2 momentos diferentes por el mismo observador a fin de obtener el error estadístico. Posteriormente, se realizó un análisis descriptivo de los datos y la comparación con la información existentes en la literatura actual. Cuando fue necesario, se utilizó análisis estadístico con la prueba t de student considerando el valor de $\mathrm{p}<0,05$ para establecer diferencias significativas.

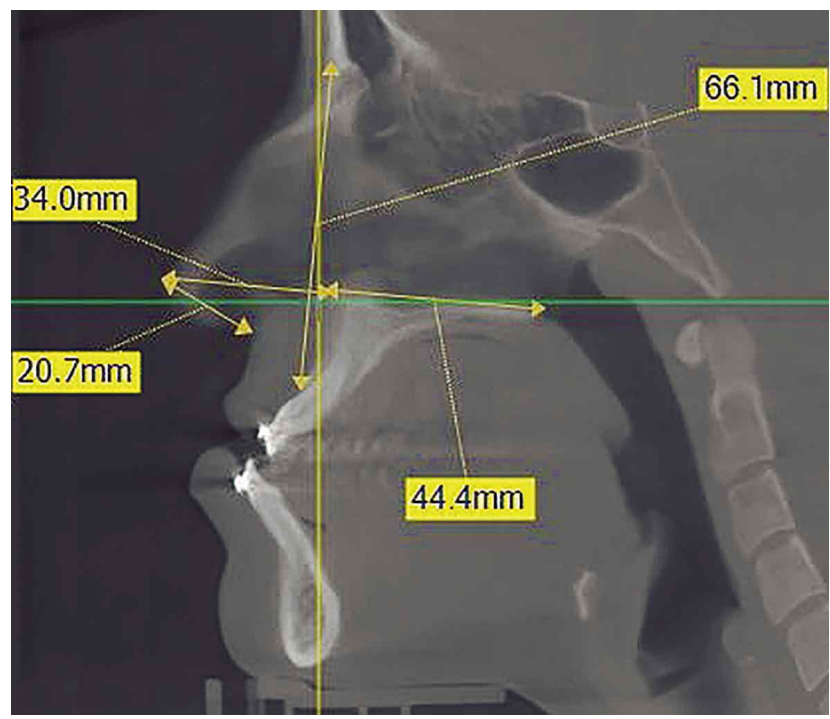

Fig. 2. Relaciones morfométricas obtenidas en imagen lateral de TC cone beam, abordando las mediciones lineales sagitales de sujetos con deformidad facial clase III.

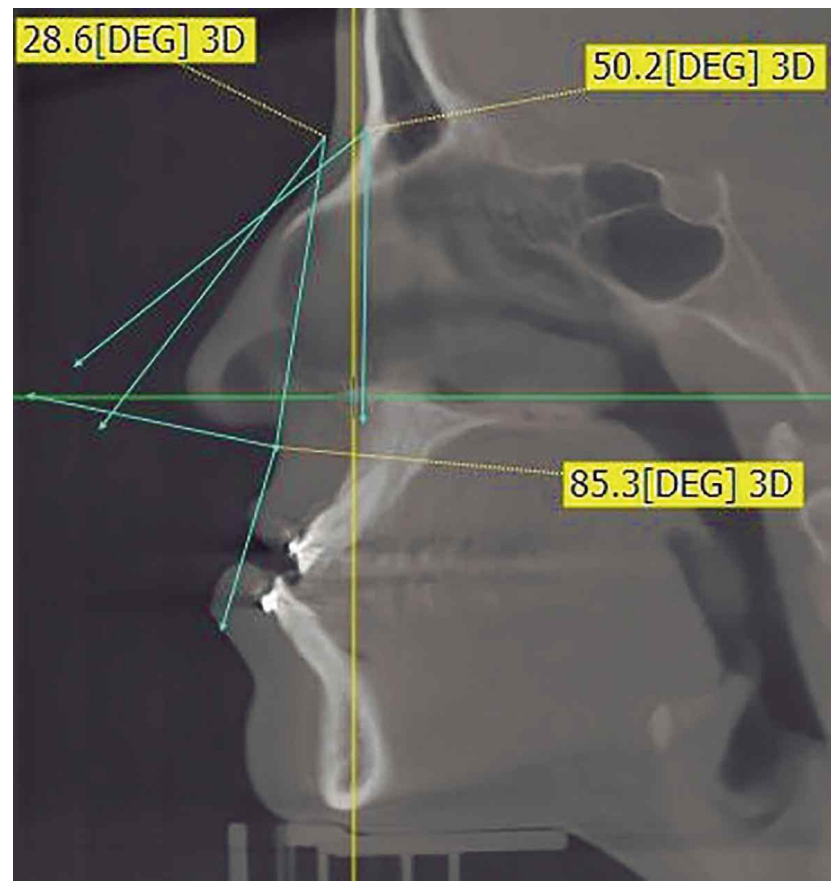

Fig. 3. Relaciones morfométricas angulares realizadas en imagen sagital de TC cone beam de individuos con deformidad facial clase III. 


\section{RESULTADOS}

El protocolo del análisis clínico facial se desarrolló sin inconvenientes. La distancia vertical entre el Nasion y el punto cervical del incisivo central $(67,08 \pm 7,19 \mathrm{~mm})$, junto con la medición del ángulo nasolabial $\left(100,76 \pm 8,08^{\circ}\right)$ fueron las que presentaron mayor variación. Sin embargo, ninguna de las evaluaciones presentó diferencias estadísticamente significativas. El ángulo nasal de tejidos
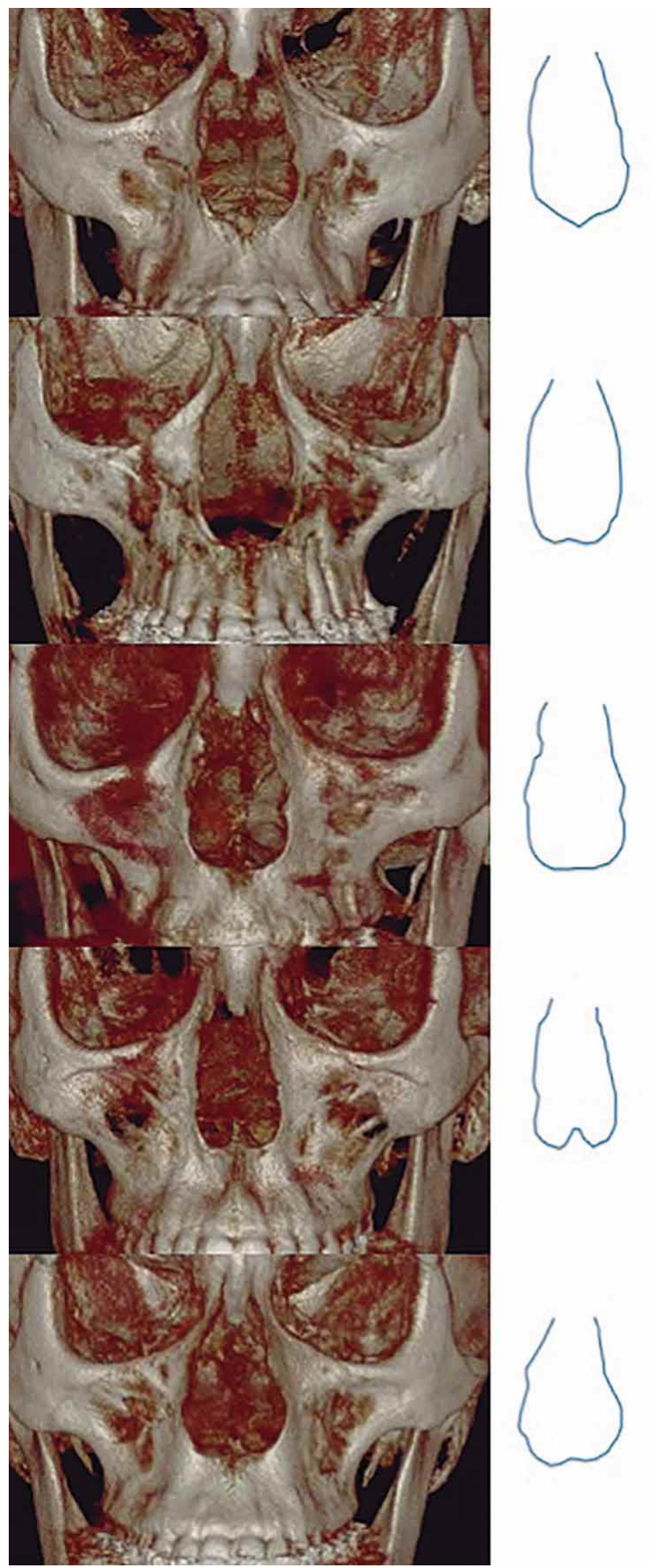

blandos y duros presentó una relación muy próxima. Los diferentes valores observados se muestran en la Tabla I.

Aunque existió una distancia de ancho nasal sin diferencias significativas entre los sujetos evaluados $(\mathrm{p}=0,46)$, se observó una amplia variabilidad de la morfología de la apertura piriforme (Fig. 4).

\section{DISCUSIÓN}

La cirugía de nariz es altamente exigente, ya que la mayoría de los pacientes que se operan solicitan una rinoplastía de tipo estética y requieren osteotomías nasales laterales, nasales dorsales y/o modificación de la punta nasal. Esto demuestra la versatilidad de los procedimientos y el papel que cumple la posición de huesos nasales su ejecución (Bagheri et al., 2012). Los casos de cirugía ortognática están vinculados a condiciones similares, donde cerca del $60 \%$ de los paciente se realiza la cirugía por motivos estéticos (Palumbo et al., 2006).

La nariz es una de las estructuras faciales con mayor variación en su forma; esta observación fue confirmada por Miles et al., (2007) quienes señalaron que las variaciones anatómicas del septo nasal son extremadamente altas, transformando la variación como una norma, más que una excepción en la anatomía de la nariz. Nuestros resultados mostraron variación de forma de la apertura piriforme; si bien no existió una cuantificación de ella, se pudo confirmar de manera cualitativa (Figura 4), con variaciones tanto en su altura como ancho superior e inferior, lo que genera disposiciones morfológicas más estrechas, ovales o triangulares de la apertura piriforme.

El ángulo nasal y la posición de la punta nasal están influenciados directamente por la posición de la ENA y conformación de los cartílagos del septo nasal, donde cerca del $55 \%$ del cartílago septal se posiciona por anterior a la ENA (Miles et al.); esta situación es relevante toda vez que deformidades faciales deben influir en la posición y forma de la nariz (Park et al., 2012) y con ello incidir en aspectos del

Fig. 4. Imágenes tomográficas de cinco sujetos con deformidad facial clase III junto al diseño de la apertura piriforme de cada uno de ellos, demostrando la variabilidad de formas obtenidas en este reparo anatómico. 
Tabla I. Distribución de mediciones de apertura piriforme y nariz obtenidas en 20 sujetos con deformidad facial clase III.

\begin{tabular}{lccc}
\hline Medición & Figura & Promedio & DE ( \pm ) \\
\hline Vertical HN & 1 & $16,02 \mathrm{~mm}$ & 3,27 \\
Ancho máximo superior apertura piriforme & 1 & $11,91 \mathrm{~mm}$ & 1,09 \\
Ancho máximo Inferior apertura piriforme & 1 & $22,28 \mathrm{~mm}$ & 1,89 \\
Distancia vertical HN-ENA & 1 & $33,27 \mathrm{~mm}$ & 3,61 \\
Distancia maxilar entre los huesos cigomáticos & 1 & $62,13 \mathrm{~mm}$ & 5,36 \\
Distancia antero-posterior maxilar & 2 & $48,76 \mathrm{~mm}$ & 4,56 \\
Vertical Nasion-Cervical incisivos centrales & 2 & $67,08 \mathrm{~mm}$ & 7,19 \\
Distancia ENA-Pronasal & 2 & $29,57 \mathrm{~mm}$ & 2,00 \\
Distancia punto A-Pronasal & 2 & $19,89 \mathrm{~mm}$ & 0,98 \\
Ángulo nasolabial & 3 & $100,76^{\circ}$ & 8,08 \\
Ángulo ENA-N-HN & 3 & $46,67^{\circ}$ & 4,91 \\
Angulo punto A-Nasion blando-Dorso nasal & 3 & $26,40^{\circ}$ & 2,19 \\
\hline
\end{tabular}

tratamiento. En este sentido, Aydil et al., (2013) detectaron variaciones importantes en los resultados de cirugías maxilomandibulares de sujetos con DF-III, probablemente asociadas a la variabilidad de la anatomía nasal. Nuestros resultados presentaron relaciones para angulaciones del dorso nasal tanto en tejidos blandos como en tejido óseo, lo cual debe considerarse en la planificación quirúrgica de avances maxilares. Por otra parte, el ancho del maxilar también se relacionó con el ancho inferior de apertura piriforme en una relación de casi 2:1, determinando que los aumentos transversales del maxilar también influirán en las características basales de la apertura piriforme. Así mismo, el ancho de apertura piriforme a nivel superior fue bastante coincidente en los sujetos evaluados. Por esto, la definición de características propias de las estructuras anatómicas nasales en sujetos con DF-III es necesaria, ya que junto a la condición anatómica anormal, elementos como las características climáticas (aire frío, caliente, seco o húmedo) influirán sobre la morfología de la apertura piriforme y nasal (Cantín et al., 2009), previa y posteriormente a la cirugía.

Begg \& Harkness identificaron que la nariz en hombres es más recta que en mujeres, aunque no se observaron diferencias significativas entre ambos sexos, mientras que la posición tampoco presentaba diferencias significativas, sin embargo, existía mayor proyección de la nariz en los hombres. Park et al., (2014) detectaron diferencias significativas en medidas de altura nasal y altura del puente nasal, presentando mayor tamaño en los hombres. A pesar de que los autores intentan conocer la influencia del crecimiento en el desarrollo de la nariz, no observaron diferencias significativas entre ambos sexos; además, una de las limitaciones de esa investigación es que no reporta el crecimiento sagital de los pacientes en términos de alteraciones del desarrollo facial tipo II o tipo III, ya que esta información se encuentra descrita en buena parte de los reportes de morfología nasal.
Los resultados de este estudio permitieron conocer algunos aspectos anatómicos de la condición nasal en sujetos con DF-III. Son escasas las medidas registradas a nivel nasal, y quizás el ángulo nasolabial sea una de las medidas más empleados en los diferentes análisis; se considera que este ángulo debe presentar $106,4 \pm 7,7^{\circ}$ en hombres y $103,5 \pm 6,8^{\circ}$ en mujeres (Legan \& Burstone, 1980); nuestros resultados mostraron un ángulo promedio de $100,76 \pm 8,08^{\circ}$. La menor medida observada en nuestra muestra fue de $85,5^{\circ}$ mientras que sólo cuatro sujetos superaron $104^{\circ}$, lo cual puede explicar la variación obtenida. La mayoría de los casos es inferior a $104^{\circ}$, lo que se relaciona con la alteración facial de clase III.

Podemos concluir que existen variaciones tanto en la altura de los huesos nasales como las características morfológicas de la apertura piriforme en sujetos con DFIII. Se deben considerar nuevas evaluaciones y parámetros en futuras investigaciones con la finalidad de que puedan ser empleadas en análisis de diagnóstico y tratamiento.

OLATE, S.; CANTÍN, M.; VÁSQUEZ, B. \& OLATE, G. Structure of the Nose in Patients with Class III Facial Deformities. Int. J. Morphol., 32(1):338-338, 2014.

SUMMARY: The aim of this study was to obtain information on some morphological aspects of the nose structure in subjects with class III facial deformities. A study was carried out by computed cone beam tomography in 20 patients between 18 and 35 years of age to measure pyriform aperture, nasal bones, dorsal nasal angle and superior lip angle; the study was realized on system software, measurement was realized in standardized conditions and the data analyzed was descriptive and with student ' $t$ ' test considering $\mathrm{p}<0.05$ for statistical significance. The results 
showed high variations in nasal bones height and pyriform aperture shape; measurement showed some variations with nasal-lip angle with values less than 104o, considered outside of the normality. In some of the measurements, anatomical variations of the nose in these subjects was clear; there were no significant statistical differences in any of the measurements in this sample. It was concluded that there are important variations in the anatomical form and new measurements should be evaluated for their use in diagnosis and treatment.

morphometry.

KEY WORDS: Facial deformity; Nose; Facial

\section{REFERENCIAS BIBLIOGRÁFICAS}

Aydil, B.; Özer, N. \& Marsan, G. Bimaxillary surgery in class III malocclusion: soft and hard tissue changes. $J$. Craniomaxillofac. Surg., 41(3):254-7, 2013.

Bagheri, S.; Khan, H. A.; Jahangirnia, A.; Rad, S. S. \& Mortazavi, H. An analysis of 101 primary cosmetic rhinoplasties. J. Oral Maxillofac. Surg., 70(4):902-9, 2012.

Begg, R. \& Harkness, M. A lateral cephalometric analysis of the adult nose. J. Oral Maxillofac. Surg., 53(11):1268-74, 1995.

Bertossi, D.; Albanese, M.; Turra, M.; Favero, V.; Nocini, P. \& Lucchese, A. Combined rhinoplasty and genioplasty: long-term follow up. JAMA Facial Plast. Surg., 15(3):192-7, 2013.

Çakir, B.; Dogan, T.; Öreroglu, A. R. \& Daniel, R. K. Rhinoplasty: surface aesthetic and surgical techniques. Aesthet. Surg. J., 33(3):363-75, 2013

Cantin, L. M.; Suazo, G. I. C.; Zavando, M. D. A. \& Smith, R. L. Sexual dimorphism determination by piriform aperture morphometric analysis in Brazilian human skulls. Int. J. Morphol., 27(2):327-31, 2009.

Legan, H. L. \& Burstone, C. J. Soft tissue cephalometric analysis for orthognathic surgery. J. Oral Surg., 38(10):744-51, 1980.

Miles, B.; Petrisor, D.; Kao, H.; Finn, R. \& Throckmorton, G. Anatomical variation of the nasal septum: analysis of 57 cadaver specimens. Otolaryngol. Head Neck Surg., 136(3):3628, 2007.

Palumbo, B.; Cassese, R.; Fusetti, S. \& Tartaro, G. P. Psychological aspects of orthognathic treatment. Minerva Stomatol., 55(12):33-42, 2006.

Park, S. B.; Yoon, J. K.; Kim, Y. I.; Hwang, D. S.; Cho, B. H. \& Son, W. S. The evaluations of the nasal morphologic changes after bimaxillary surgery in skeletal class III malocclusion by using the superimposition of cone-beam computed tomography (CBCT) volumes. J. Craniomaxillofac. Surg., 40(4):e87-92, 2012.

Park, S. W.; Choi, J.; Park, H. O.; Lim, Y. S.; Lee, K. S.; Kim, N. G. \& Kim, J. S. Are Gender Differences in External Noses Caused by Differences in Nasal Septal Growth? J. Craniomaxillofac. Surg., doi: 10.1016/j.jcms.2014.01.045, 2014.

Seah, T. E.; Bellis, H. \& Ilankovan, V. Orthognathic patients with nasal deformities: case for simultaneous orthognathic surgery and rhinoplasty. Br. J. Oral Maxillofac. Surg., 50(1):55-9, 2012.

Dirección para Correspondencia:

Prof. Dr. Sergio Olate

División de Cirugía Oral y Maxilofacial

Universidad de La Frontera

Claro Solar 115, Oficina 414-A

Temuco

CHILE

Email: sergio.olate@ufrontera.cl

Recibido : 21-12-2013

Aceptado: 19-01-2014 